\title{
The Stokes Phenomenon and Some Applications
}

\author{
Marius VAN DER PUT \\ University of Groningen, Department of Mathematics, \\ P.O. Box 407, 9700 AK Groningen, The Netherlands \\ E-mail: mvdput@math.rug.nl \\ Received January 21, 2015, in final form April 21, 2015; Published online May 01, 2015 \\ http://dx.doi.org/10.3842/SIGMA.2015.036
}

\begin{abstract}
Multisummation provides a transparent description of Stokes matrices which is reviewed here together with some applications. Examples of moduli spaces for Stokes matrices are computed and discussed. A moduli space for a third Painlevé equation is made explicit. It is shown that the monodromy identity, relating the topological monodromy and Stokes matrices, is useful for some quantum differential equations and for confluent generalized hypergeometric equations.
\end{abstract}

Key words: Stokes matrices; moduli space for linear connections; quantum differential equations; Painlevé equations

2010 Mathematics Subject Classification: 14D20; 34M40; 34M55; 53D45

\section{Introduction}

Consider a linear differential equation in matrix form $y^{\prime}+A y=0$, where the entries of the matrix $A$ are meromorphic functions defined in a neighbourhood of, say, $z=\infty$ in the complex plane. A formal or symbolic solution can be lifted to an actual solution in a sector at $z=\infty$, having the formal solution as asymptotic behavior.

In 1857, G.G. Stokes observed, while working in the middle of the night and not long before getting married, the phenomenon that this lifting depends on the direction of the sector at $z=\infty$ (see [23, 24] for more details).

This is the starting point of the long history of the asymptotic theory of singularities of differential equations. The theory of multisummation is the work of many mathematicians such as W. Balser, B.L.J. Braaksma, J. Écalle, W.B. Jurkat, D. Lutz, M. Loday-Richaud, B. Malgrange, J. Martinet, J.-P. Ramis, Y. Sibuya (see [19, 20] for excellent bibliographies and references and also [30] for some details).

This paper reviews the transparent description of the Stokes phenomenon made possible by multisummation and some applications of this, namely:

a) moduli spaces of linear differential equations,

b) quantum differential equations and confluent generalized hypergeometric equations,

c) isomonodromy, the Painlevé equations and Okamoto-Painlevé spaces.

Moreover the paper presents new ideas on moduli spaces for the Stokes phenomenon and the use of the monodromy identity.

The first section is written for the convenience of the reader. It is a review of a large part of [30] and has as new part Section 2.5. The aim is to describe clearly and concisely the theory and results while bypassing technical details and proofs.

${ }^{\star}$ This paper is a contribution to the Special Issue on Algebraic Methods in Dynamical Systems. The full collection is available at http://www.emis.de/journals/SIGMA/AMDS2014.html 
One considers a singular matrix differential equation $y^{\prime}+A y=0$ at $z=\infty$ and recalls its formal classification, the definition of the Stokes matrices and the analytic classification. The theory is illustrated in Section 2.5 by the confluent generalized hypergeometric equation ${ }_{p} D_{q}$. We rediscover results from $[11,22]$ as application of the monodromy identity. In the second section moduli spaces for Stokes maps are discussed. In the new part Section 3.2 and especially Proposition 2, it is shown that totally ramified equations have very interesting Stokes matrices. Quantum differential equations coming from Fano varieties are studied in Section 4. Again the computability of Stokes matrices is the theme. Certain moduli spaces, namely OkamotoPainlevé spaces, corresponding to Painlevé equations are discussed in Section 5, including an explicit calculation of a monodromy space for $\mathrm{P}_{\mathrm{III}}\left(\mathrm{D}_{7}\right)$.

\section{Formal and analytic classification, Stokes maps}

\subsection{Terminology and notation}

Let $k$ be a differential field, i.e., a field with a map $f \mapsto f^{\prime}$ (called a derivation) satisfying $(f+g)^{\prime}=f^{\prime}+g^{\prime}$ and $(f g)^{\prime}=f^{\prime} g+f g^{\prime}$. The field of constants of $k$ is $\left\{f \in k \mid f^{\prime}=0\right\}$. In this paper we suppose that the field of constants of $k$ is $\mathbb{C}$ and that $k \neq \mathbb{C}$.

The most important differential fields that we will meet are $\mathbb{C}(z), \widehat{K}:=\mathbb{C}\left(\left(z^{-1}\right)\right)$ and $K:=$ $\mathbb{C}\left(\left\{z^{-1}\right\}\right)$, i.e., the field of the rational functions in $z$, the formal Laurent series in $z^{-1}$ and the field of the convergent Laurent series in $z^{-1}$. The latter is the field of germs of meromorphic functions at $z=\infty$. In all cases the differentiation is $f \mapsto f^{\prime}=\frac{d f}{d z}$ (sometimes replaced by $f \mapsto \delta(f):=z \frac{d f}{d z}$ in order to make the formulas nicer).

A matrix differential equation $y^{\prime}+A y=0$ with $A$ a $d \times d$-matrix with coordinates in $k$ gives rise to the operator $\partial:=\frac{d}{d z}+A$ of $k^{d}$, where $\frac{d}{d z}$ acts coordinatewise on $k^{d}$ and $A$ is the matrix of a $k$-linear map $k^{d} \rightarrow k^{d}$. Write now $M=(M, \partial)$ for $k^{d}$ and the operator $\partial$. Then this object is a differential module.

Indeed, a differential module over $k$ is a finite dimensional $k$-vector space $M$ equipped with an additive map $\partial: M \rightarrow M$ satisfying $\partial(f m)=f^{\prime} m+f \partial(m)$ for any $f \in k$ and $m \in M$. If one fixes a basis of $M$ over $k$, then $M$ is identified with $k^{d}$ (with $d=\operatorname{dim} M$ ) and the operator $\partial$ is identified with $\frac{d}{d z}+A$. Here $A$ is the matrix of $\partial$ with respect to the given basis of $M$.

Thus a differential module is "a matrix differential equation where the basis is forgotten" and a matrix differential equation is the same as a differential module with a given basis.

We will use differential operators, i.e., elements of the skew polynomial ring $k[\partial]$ (where $\partial$ stands for $\frac{d}{d z}$ ) defined by the rule $\partial f=f \partial+f^{\prime}$. Instead of $\partial$ we sometimes use $\delta:=z \partial$. Then $\delta f=f \delta+\delta(f)$ (in particular $\delta z=z \delta+z)$.

Let $M$ be a differential module. The ring $k[\partial]$ acts from the left on $M$. For any element $e \in M$, there is a monic operator $L \in k[\partial]$ of smallest degree such that $L e=0$. The element $e$ is called cyclic if $L$ has degree $d=\operatorname{dim} M$. Cyclic elements $e$ exist [30, $\S \S 2.10$ and 2.11]. The corresponding operator $L$ is identified with a scalar differential equation and $L$ determines $M$.

In practice one switches between differential modules, matrix differential equations, scalar differential equations and differential operators.

\subsection{Classification of differential modules over $\widehat{K}:=\mathbb{C}\left(\left(z^{-1}\right)\right)$}

The classification of a matrix differential equation $z \frac{d}{d z}+A$ over $\widehat{K}$ (note that we prefer here $z \frac{d}{d z}$ ) goes back to G. Birkhoff and H.L. Turritin. This classification is somewhat similar to the Jordan normal of a matrix. However it is more subtle since $z \frac{d}{d z}+A$ is linear over $\mathbb{C}$ and is not linear over $\widehat{K}$. 
We prefer to work "basis free" with a differential module $M$ and classify $M$ by its solution space $V$ with additional data forming a tuple $\left(V,\left\{V_{q}\right\}, \gamma\right)$.

If $\operatorname{dim} M=d$, then we want the solution space, i.e., the elements $w$ with $\delta w=0$ to be a $\mathbb{C}$-vector space of dimension $d$. Now we write $\delta: M \rightarrow M$ instead of $\partial$ because $\frac{d}{d z}$ is replaced by $z \frac{d}{d z}$.

In general $\{m \in M \mid \delta(m)=0\}$ is a vector space over $\mathbb{C}$ with dimension $<d$. Thus we enlarge $\widehat{K}$ to a suitable differential ring $U$ and consider $\left\{w \in U \otimes_{\widehat{K}} M \mid \delta w=0\right\}$.

This differential ring $U$ (the universal Picard-Vessiot ring for $\widehat{K}$ ) is built as follows. We need a linear space of "eigenvalues" $\mathcal{Q}:=\cup_{m \geq 1} z^{1 / m} \mathbb{C}\left[z^{1 / m}\right]$ and symbols $z^{\lambda}$ with $\lambda \in \mathbb{C}, \log z, e(q)$ with $q \in \mathcal{Q}$. The relations are $z^{a+b}=z^{a} \cdot z^{b}, z^{1}=z \in \widehat{K}, e\left(q_{1}+q_{2}\right)=e\left(q_{1}\right) \cdot e\left(q_{2}\right), e(0)=1$. And we define their derivatives by the formulas $\left(z^{a}\right)^{\prime}=a z^{a}, \log (z)^{\prime}=1, e(q)^{\prime}=q e(q)$ (we note that 'stands for $z \frac{d}{d z}$ and that the interpretation of $e(q)$ is $\left.e^{\int q \frac{d z}{z}}\right)$.

This universal Picard-Vessiot ring is $U:=\widehat{K}\left[\left\{z^{\lambda}\right\}_{\lambda \in \mathbb{C}}, \log (z),\{e(q)\}_{q \in \mathcal{Q}}\right]$. This ring is a direct sum $U=\oplus_{q \in \mathcal{Q}} U_{q}$ and $U_{q}:=e(q) \widehat{K}\left[\left\{z^{\lambda}\right\}_{\lambda \in \mathbb{C}}, \log (z)\right]$.

The Galois group of the algebraic closure $\cup_{m>1} \mathbb{C}\left(\left(z^{-1 / m}\right)\right)$ of $\widehat{K}$ is $\cong \widehat{\mathbb{Z}}$ and is topologically generated by the element $\gamma$ given by $\gamma z^{\lambda}=e^{2 \pi i \lambda} z^{\lambda}$ for all $\lambda \in \mathbb{Q}$. The algebraic closure of $\widehat{K}$ lies in $U$. One extends $\gamma$ to a differential automorphism of $U$ by the following formulas (corresponding to the interpretation of the symbols) $\gamma z^{a}=e^{2 \pi i a} z^{a}$ for all $a \in \mathbb{C}, \gamma \log (z)=$ $2 \pi i+\log (z), \gamma e(q)=e(\gamma q)$.

For every differential module $M$ over $\widehat{K}$, its solution space, defined as $V:=\operatorname{ker}(\delta, U \otimes M)$ has "all solutions" in the sense that $\operatorname{dim}_{\mathbb{C}} V=\operatorname{dim}_{\widehat{K}} M$ and the canonical map $U \otimes_{\mathbb{C}} V \rightarrow U \otimes_{\widehat{K}} M$ is an isomorphism.

Put $V_{q}:=\operatorname{ker}\left(\delta, U_{q} \otimes M\right)$. Then $V=\oplus_{q} V_{q}$ is a decomposition of the solution space. Further the action of $\gamma$ on $U$ induces a $\gamma \in \operatorname{GL}(V)$ such that $\gamma V_{q}=V_{\gamma q}$ for all $q$.

Theorem 1 (formal classification [30, Proposition 3.30]). The functor $M \mapsto\left(V,\left\{V_{q}\right\}, \gamma\right)$ is an equivalence of the Tannakian categories of the differential modules over $\widehat{K}$ and the category of the tuples $\left(V,\left\{V_{q}\right\}, \gamma\right)$.

The category of the tuples $\left(V,\left\{V_{q}\right\}, \gamma\right)$ is denoted by $\mathrm{Gr}_{1}$ in [30, p. 76]. The "Tannakian" property of the functor of the theorem means that it commutes with all constructions of linear algebra, including tensor products, applied to modules.

Suppose that $M$ induces the tuple $\left(V,\left\{V_{q}\right\}, \gamma\right)$. Then $q$ is called an eigenvalue of $M$ if $V_{q} \neq 0$. The Katz invariant of $M$ is the maximum of the degrees in $z$ of the eigenvalues $q$ of $M$. Clearly, the Katz invariant is a non negative rational number which measures the singularity. In particular, the Katz invariant is zero if and only if the module $M$ is regular singular.

Further the map $\gamma \in \mathrm{GL}(V)$ is called the formal monodromy of $M$. Section 2.3 illustrates the computation of the tuple $\left(V,\left\{V_{q}\right\}, \gamma\right)$.

\subsection{The confluent generalized hypergeometric equation}

Consider the confluent generalized hypergeometric equation in operator form

$$
{ }_{p} D_{q}=(-1)^{q-p} z \prod_{j=1}^{p}\left(\delta+\mu_{j}\right)-\prod_{j=1}^{q}\left(\delta+\nu_{j}-1\right) \quad \text { with } \quad \delta=z \frac{d}{d z} .
$$

We assume that $1 \leq p<q$ and that the complex parameters $\mu_{j}, \nu_{j}$ are such that $\mu_{1}, \ldots, \mu_{p}$ are distinct modulo $\mathbb{Z}$. We regard the operator ${ }_{p} D_{q}$ as element of $\widehat{K}[\delta]$.

Its Newton polygon has two slopes 0 and $\frac{1}{q-p}$. The operator has then for each ordering of the slopes a unique factorization (compare [30, Theorem 3.8]). The two decompositions are

$$
{ }_{p} D_{q}=-\left(\delta^{p}+a_{p-1} \delta^{p-1}+\cdots+a_{0}\right)\left(\delta^{q-p}+b_{q-p-1} \delta^{q-p-1}+\cdots+b_{1} \delta-(-1)^{q-p} z+b_{0}\right)
$$


with all $a_{j}, b_{j} \in \mathbb{C}\left[\left[z^{-1}\right]\right]$. The two factors almost commute and there is a factorization in the opposite ordering of the slopes

$$
{ }_{p} D_{q}=-\left(\delta^{q-p}+b_{q-p-1}^{*} \delta^{q-p-1}+\cdots+b_{1}^{*} \delta-(-1)^{q-p} z+b_{0}^{*}\right)\left(\delta^{p}+a_{p-1}^{*} \delta^{p-1}+\cdots+a_{0}^{*}\right)
$$

with all $a_{j}^{*}, b_{j}^{*} \in \mathbb{C}\left[\left[z^{-1}\right]\right]$.

From these factorizations one can read off the solution space. The term $\left(\delta^{p}+a_{p-1}^{*} \delta^{p-1}+\cdots+\right.$ $\left.a_{1}^{*} \delta+a_{0}^{*}\right)$ is equivalent to $\prod_{j=1}^{p}\left(\delta+\mu_{j}\right)$ ('equivalent' means that the differential modules over $\widehat{K}$ defined by the two operators are isomorphic). This yields solutions $f_{j}:=z^{-\mu_{j}}$ for $j=1, \ldots, p$. They form a basis of the $\mathbb{C}$-vector space $V_{0}$ with eigenvalue $q_{0}=0$. The action of the formal monodromy $\gamma$ on $V_{0}$ is $\gamma\left(f_{j}\right)=e^{-2 \pi i \mu_{j}} f_{j}$.

The term $\left(\delta^{q-p}+b_{q-p-1} \delta^{q-p-1}+\cdots+b_{1} \delta-(-1)^{q-p} z+b_{0}\right)$ is equivalent to $\delta^{\sigma}-z$ (up to a sign) with $\sigma:=q-p$. This operator factors over $\widehat{K}\left(z^{1 / \sigma}\right)$ and one finds the eigenvalues $q_{1}=z^{1 / \sigma}$, $q_{2}=\zeta z^{1 / \sigma}, \ldots, q_{\sigma}=\zeta^{\sigma-1} z^{1 / \sigma}$ with $\zeta=e^{2 \pi i / \sigma}$.

Now we can describe the solution space $V$ of ${ }_{p} D_{q}$ :

$$
V=V_{0} \oplus V_{q_{1}} \oplus \cdots \oplus V_{q_{\sigma}}
$$

where $V_{0}$ has a basis $f_{1}, \ldots, f_{p}$ with $\gamma f_{j}=e^{-2 \pi i \mu_{j}} f_{j}$. Choose a basis $e_{1}$ of the 1-dimensional space $V_{q_{1}}$. Put $e_{2}:=\gamma e_{1}, e_{3}:=\gamma e_{2}, \ldots, e_{\sigma}:=\gamma e_{\sigma-1}$. Then $V_{q_{j}}=\mathbb{C} e_{j}$ for $j=1, \ldots, \sigma$. Finally $\gamma e_{\sigma}=e^{2 \pi i \lambda} e_{1}$ with $\lambda:=\frac{1}{2}(\sigma+1)+\sum_{j=1}^{p} \mu_{j}-\sum_{j=1}^{q} \nu_{j}$. This follows from a computation of $\gamma$ on $f_{1} \wedge \cdots \wedge f_{p} \wedge e_{1} \wedge \cdots \wedge e_{\sigma}$ and the determinant $\delta+\sum_{j=1}^{q}\left(v_{j}-1\right)$ of ${ }_{p} D_{q}$.

The above coincides with the formula of the formal monodromy in [22, p. 373]. In [11, 22] an explicit basis of formal or symbolic solutions of ${ }_{p} D_{q}$ is constructed and the computation of the formal monodromy and, later on, of the Stokes matrices is with respect to this basis. Our basis $f_{1}, \ldots, f_{p}, e_{1}, \ldots, e_{\sigma}$ is not unique. More precisely, the above tuple has a non trivial automorphism group $G \cong\left(\mathbb{C}^{*}\right)^{p+1}$. The elements of $G$ are given by $f_{j} \mapsto \alpha_{j} f_{j}$ for $j=1, \ldots, p$ and $e_{j} \mapsto \alpha_{p+1} e_{j}$ for $j=1, \ldots, \sigma$ (and $\left.\left(\alpha_{1}, \ldots, \alpha_{p+1}\right) \in G\right)$. We will see that the group $G / \mathbb{C}^{*}$ acts non trivially on the entries of the Stokes maps (see also Proposition 2).

\subsection{Stokes maps and the analytic classification}

Let $M$ be a differential module over $K$. Then $\widehat{K} \otimes M$ is a differential module over $\widehat{K}$ and induces a tuple $\left(V,\left\{V_{q}\right\}, \gamma\right)$. For two eigenvalues $q, \tilde{q}$ of $\widehat{K} \otimes M$ one considers special directions $e^{2 \pi i d}$, $d \in \mathbb{R}$, called singular for the difference $q-\tilde{q}$. Those are the $d$ such that $e^{\int(q-\tilde{q}) \frac{d z}{z}}$ (this is the solution of $\left.y^{\prime}=(q-\tilde{q}) y\right)$ has maximal descent to zero for $z:=r e^{2 \pi i d}$ and $r>0, r \rightarrow 0$.

The tuple $\left(V,\left\{V_{q}\right\}, \gamma\right)$ is the formal classification of $M$, i.e., the classification of $\widehat{K} \otimes_{K} M$. Now we consider the classification of $M$ itself. In the sequel, we use multisummation as a black box. It is a technical extension of the classical Borel summation of certain divergent power series. We refer to $[2,3,30]$ for details.

However, we will not need the precise formulation of the multisum in a certain direction $d$. This is rather technical [30, Definition 7.46].

The sloppy definition and result is as follows. For a direction $d$ which is not singular, one considers a certain sector $S(d)$ at $z=\infty$ around $d$ (or more precisely, a sequence of nested sectors). There is a unique $\mathbb{C}$-linear map, the multisum

$\operatorname{sum}_{d}: V \rightarrow$ the space of the solutions of $M$ on the sector $S(d)$. 
It has the property that for any $v \in V$, the $\operatorname{sum}_{d}(v)$ has asymptotic expansion $v$ on the prescribed sector (or more precisely, a sequence of asymptotic properties on the nested sequence of sectors).

We note that the uniqueness of $\mathrm{sum}_{d}$ is the important issue of multisummation. This is a much stronger result than the more classical "Main asymptotic existence theorem" [30, Theorem 7.10].

For a singular direction $d$ one takes real numbers $d^{-}<d<d^{+}$close to $d$ and defines the Stokes map $\mathrm{St}_{d} \in \mathrm{GL}(V)$ by $\operatorname{sum}_{d^{+}}=\operatorname{sum}_{d^{-}} \circ \mathrm{St}_{d}$. To $M$ we associate the tuple $\left(V,\left\{V_{q}\right\}, \gamma,\left\{\mathrm{St}_{d}\right\}_{d \in \mathbb{R}}\right)$.

One can show [30, Theorem 8.13 and Remark 8.14] that this tuple has the additional properties:

(*) $\mathrm{St}_{d}$ has the form id $+\sum_{d \text { singular for } q-\tilde{q}} \operatorname{Hom}\left(V_{q}, V_{\tilde{q}}\right)$. Here, every $\ell \in \operatorname{Hom}\left(V_{q}, V_{\tilde{q}}\right)$ is seen as an element of $\operatorname{End}(V)$ by the sequence of maps $V \stackrel{\text { projection }}{\rightarrow} V_{q} \stackrel{\ell}{\rightarrow} V_{\tilde{q}} \stackrel{\text { inclusion }}{\rightarrow} V$.

(**) $\gamma^{-1} \mathrm{St}_{d} \gamma=\mathrm{St}_{d+1}$.

One considers the category of the tuples with the properties $(*)$ and $(* *)$. This category of finite dimensional complex vector spaces with this additional "linear algebra structure" is denoted by $\mathrm{Gr}_{2}$ in $[30, \S 9.2]$. It is a Tannakian category.

Theorem 2 (the analytic classification [30, Theorem 9.11]). The functor $M \mapsto\left(V,\left\{V_{q}\right\}, \gamma,\left\{\mathrm{St}_{d}\right\}\right)$ is an equivalence of the Tannakian categories of the differential modules over $K$ and the above category of the tuples $\left(V,\left\{V_{q}\right\}, \gamma,\left\{\mathrm{St}_{d}\right\}\right)$ satisfying $(*)$ and $(* *)$.

The above theorem is, in contrast with the formal classification, a deep and final result in the asymptotic theory of linear differential equations.

The irregularity of Malgrange, $\operatorname{irr}(M)$ of the differential module $M$ is defined by $\operatorname{irr}(M)=$ $\sum_{q \neq \tilde{q}} \operatorname{deg}_{z}(q-\tilde{q}) \cdot \operatorname{dim} V_{q} \cdot \operatorname{dim} V_{\tilde{q}}$. One observes that the dimension of the space of all possibilities for Stokes maps with a fixed formal tuple is equal to $\operatorname{irr}(M)$ (see also Section 3). A useful result, obtained by the above description of the Stokes maps, is the following.

Proposition 1 (the monodromy identity [30, Proposition 8.12]). The topological monodromy of $M$ is conjugated to $\gamma \mathrm{St}_{d_{s}} \cdots \mathrm{St}_{d_{1}} \in \mathrm{GL}(V)$, where $0 \leq d_{1}<\cdots<d_{s}<1$ are the singular directions of $M$.

One cannot claim that the topological monodromy is equal to this product since one has to identify $V$ with the local solution space at a point near $z=\infty$ and that can be done in many ways.

\subsection{Stokes matrices for ${ }_{p} D_{q}$}

The main observation is the following. The monodromy identity yields complete formulas for the Stokes matrices of ${ }_{p} D_{q}$ if we are allowed to choose a suitable basis of $V$. In this way we rediscover the formulas for the Stokes matrices of [11, 22]. More precisely, by choosing multiples of the basis $f_{1}, \ldots, f_{p}$, we can normalize $p$ entries of the Stokes maps to be 1 . The others are then determined by the monodromy identity. This works, according to Proposition 2, under the assumption that $\mu_{1}, \ldots, \mu_{p}$ are distinct modulo $\mathbb{Z}$ and that the equation is irreducible.

We note that the differential Galois group of ${ }_{p} D_{q}$ is in fact the differential Galois group of ${ }_{p} D_{q}$ as equation over the field of convergent Laurent series $K$. This group does not depend on the choice of multiples of $f_{1}, \ldots, f_{p}$. For the rather involved computation of this differential Galois group, see [11, 22], the knowledge of the formal classification and the characteristic polynomial of the topological monodromy at $z=0$ (or equivalently at $z=\infty$ ) suffice. We illustrate this by the easy example ${ }_{1} D_{3}=z(\delta+\mu)-\prod_{j=1}^{3}\left(\delta+\nu_{j}-1\right)$. 
The formal solution space is the direct sum of three 1-dimensional spaces $V_{0} \oplus V_{z^{1 / 2}} \oplus V_{-z^{1 / 2}}$ with basis $f_{1}, e_{1}, e_{2}$. There is only one singular direction in the interval $[0,1)$, namely $d=0$.

The topological monodromy at $z=0$ is that of the operator $\prod_{j=1}^{3}\left(\delta+\nu_{j}-1\right)$ and has eigenvalues $e^{-2 \pi i \nu_{j}}, j=1,2,3$.

The formal monodromy is $\gamma\left(f_{1}\right)=e^{-2 \pi i \mu} f_{1}, \gamma e_{1}=e_{2}$ and $\gamma\left(e_{2}\right)=e^{-2 \pi i \lambda} e_{1}$. The product of the formal monodromy and the unique Stokes matrix in the interval $[0,1$ ) (its form is described in Theorem 2) is

$$
\left(\begin{array}{ccc}
d_{1} & 0 & 0 \\
0 & 0 & d_{2} \\
0 & 1 & 0
\end{array}\right)\left(\begin{array}{ccc}
1 & x_{1,0} & 0 \\
0 & 1 & 0 \\
x_{0,2} & x_{1,2} & 1
\end{array}\right)=\left(\begin{array}{ccc}
d_{1} & d_{1} x_{1,0} & 0 \\
d_{2} x_{0,2} & d_{2} x_{1,2} & d_{2} \\
0 & 1 & 0
\end{array}\right)
$$

where $d_{1}=e^{-2 \pi i \mu}, d_{2}=e^{-2 \pi i \lambda}, \lambda=\frac{3}{2}+\mu-\sum \nu_{j}$. Its characteristic polynomial $T^{3}-\left(d_{1}+\right.$ $\left.d_{2} x_{1,2}\right) T^{2}-\left(d_{1} d_{2} x_{1,0} x_{0,2}-d_{2}\right) T+d_{1} d_{2}$ coincides with $\prod_{j=1}^{3}\left(T-e^{2 \pi i v_{j}}\right)$. Choose $x_{1,0}=1$. Then all Stokes matrices are determined.

The exceptional case $x_{1,0} x_{0,2}=0$ cannot be handled in this way. Using J.-P. Ramis result [30, Theorem 8.10] and [6, Théorème 4.10] that the differential Galois group is generated as algebraic group by the formal monodromy, the exponential torus and the Stokes matrices, one concludes that $x_{1,0}=0$ or $x_{0,2}=0$ implies that the equation is reducible (compare the proof of Proposition 2). See [11] for a complete description of all cases and all differential Galois groups. We remark that the monodromy identity for ${ }_{p} D_{q}$ is explicitly present in [11].

\section{Moduli spaces for the Stokes data}

For given formal data $F:=\left(V,\left\{V_{q}\right\}, \gamma\right)$ at $z=\infty$, there exists a unique differential module $N$ over $K=\mathbb{C}\left(\left\{z^{-1}\right\}\right)$ with these formal data and with trivial Stokes matrices. One considers differential modules $M$ which have formal classification $F$. The set of isomorphism classes of these modules does not have a good algebraic structure since $F$ has, in general, automorphisms.

D.G. Babbitt and V.S. Varadarajan [1] consider instead pairs $(M, \phi)$ of a differential module $M$ over $K$ and an isomorphism $\phi: \widehat{K} \otimes M \rightarrow \widehat{K} \otimes N$. Two pairs $\left(M_{j}, \phi_{j}\right), j=1,2$, are equivalent if there exists an isomorphism $\alpha: M_{1} \rightarrow M_{2}$ such that $\phi_{2} \circ \alpha=\phi_{1}$. The set Stokesmoduli $(F)$ of equivalence classes of pairs $(M, \phi)$ has been given a natural structure of complex algebraic variety. Babbitt and Varadarajan prove that $\operatorname{Stokesmoduli}(F)$ is isomorphic to the affine space $\mathbb{A}_{\mathbb{C}}^{m}$, where $m=\sum_{i \neq j} \operatorname{dim} V_{q_{i}} \cdot \operatorname{dim} V_{q_{j}} \cdot \operatorname{deg}_{z}\left(q_{i}-q_{j}\right)$. The $\left\{q_{i}\right\}$ are the eigenvalues of $M$ and $m$ is the irregularity of $F$, in the terminology of B. Malgrange.

This result of is in complete agreement with the above description of the Stokes matrices $\left\{\mathrm{St}_{d}\right\}$ (defined by multisummation). Thus Stokesmoduli $(F)$ is the moduli space for the possible Stokes matrices for fixed formal data $F=\left(V,\left\{V_{q}\right\}, \gamma\right)$.

However, there is, in general, no universal family of differential modules parametrized by $\operatorname{Stokesmoduli}(F) \cong \operatorname{Spec}\left(\mathbb{C}\left[x_{1}, \ldots, x_{m}\right]\right)$. In other words, Stokesmoduli $(F)$ is, in general, not a fine moduli space for the above family of differential modules.

Indeed, suppose that such a family $\left\{M_{\xi} \mid \xi \in \operatorname{Stokesmoduli}(F)\right\}$ of differential modules over $K=\mathbb{C}\left(\left\{z^{-1}\right\}\right)$ exists. This family is represented by a matrix differential operator $z \frac{d}{d z}+A$ in the variable $z$ and with entries in, say, $K\left(x_{1}, \ldots, x_{m}\right)$. The monodromy identity shows that the eigenvalues of the topological monodromy are algebraic over this field. A logarithm of the topological monodromy is computable from $z \frac{d}{d z}+A$ and has again entries in $K\left(x_{1}, \ldots, x_{m}\right)$. This is, in general, not possible. See Section 3.1 for a concrete case. 
In order to produce a fine moduli space one replaces the differential module $M$ over $K=$ $\mathbb{C}\left(\left\{z^{-1}\right\}\right)$ by a tuple $(\mathcal{M}, \nabla, \phi)$. Here $(\mathcal{M}, \nabla)$ is a connection on the projective line $\mathbb{P}^{1}$ over $\mathbb{C}$ which has two singular points 0 and $\infty$. The point $z=0$ is supposed to be regular singular. Further $\phi$ is an isomorphism of the formal completion of the connection at $z=\infty$ (i.e., $\mathcal{M}_{\infty} \otimes$ $\mathbb{C}\left[\left[z^{-1}\right]\right]$ ) with a prescribed object $\nabla: N_{0} \rightarrow N_{0} \otimes z^{k} \mathbb{C}\left[\left[z^{-1}\right]\right]$ (for suitable $k$, see below).

This prescribed object is the following. Let $N$ denote the differential module over $\mathbb{C}\left(\left(z^{-1}\right)\right)$ corresponding to the given $F=\left(V,\left\{V_{q}\right\}, \gamma\right)$. Then $N_{0} \subset N$ is the $\mathbb{C}\left[\left[z^{-1}\right]\right]$-submodule of $N$, generated by a basis of $N$ over $\mathbb{C}\left(\left(z^{-1}\right)\right)$ (i.e., $N_{0}$ is a lattice in $\left.N\right)$. There are many choices for $N_{0}$ and in principle the choice is not important. However we choose a standard lattice $N_{0}$ (see [30, $\S \S 12.4$ and 12.5]) in order to make explicit computation easier. The given differential operator $\nabla: N \rightarrow N$ maps $N_{0}$ into $z^{k} N_{0} \subset N$ for a certain integer $k \geq 0$. The choice of $k$ is irrelevant for the construction.

According to a theorem of Birkhoff [30, Lemma 12.1], this "spreading out of $M$ " exists. We will make the assumption that $\mathcal{M}$ is a free vector bundle on $\mathbb{P}^{1}$. The above description leads to a fine moduli space $\operatorname{Mod}(F)$.

Let $\operatorname{Stm}: \operatorname{Mod}(F) \rightarrow \operatorname{Stokesmoduli}(F)$, denote the map which associates to a tuple $(\mathcal{M}, \nabla, \phi)$, belonging to $\operatorname{Mod}(F)$, its set of Stokes matrices. Known results are:

Theorem 3 ([30, $\S \S 12.11,12.17,12.19,12.20])$.

(a) $\operatorname{Mod}(F)$ is isomorphic to the affine space $\mathbb{A}_{\mathbb{C}}^{m}$.

(b) Stm is analytic and has an open dense image.

(c) The generic fibre of Stm is a discrete infinite set and can be interpreted as a set of logarithms of the topological monodromy.

Comments. The proof of (a) is complicated and the result itself is somewhat amazing.

(b) follows from the observations: If the topological monodromy of $M$ is semi-simple, then a tuple $(\nabla, \mathcal{M}, \phi)$ with free $\mathcal{M}$ exists. Moreover semi-simplicity is an open property.

(c) follows from the construction of "spreading out". One needs a logarithm of the topological monodromy in order to construct the connection $(\mathcal{M}, \nabla)$ on $\mathbb{P}^{1}$ from the differential module over $K=\mathbb{C}\left(\left\{z^{-1}\right\}\right)$.

A precise description of the fibres seems rather difficult. Moreover, a better moduli space, replacing $\operatorname{Mod}(F)$, which does not require the vector bundle $\mathcal{M}$ to be free, should be constructed.

\subsection{Example: unramified cases}

$F$ is defined by $V=V_{\lambda_{1} z} \oplus \cdots \oplus V_{\lambda_{n} z}$, where each $V_{\lambda_{j} z}$ has dimension one and the $\lambda_{1}, \ldots, \lambda_{n} \in \mathbb{C}$ are distinct. Further $\gamma$ is the identity. Then $N_{0}$ can be given by the differential operator $\delta+z \cdot \operatorname{diag}\left(\lambda_{1}, \ldots, \lambda_{n}\right)$. Clearly $\operatorname{Stokesmoduli}(F) \cong \mathbb{A}_{\mathcal{C}}^{n(n-1)}$.

The universal family is $\delta+z \cdot \operatorname{diag}\left(\lambda_{1}, \ldots, \lambda_{n}\right)+\left(T_{i, j}\right)$, where for notational convenience $T_{i, i}=0$ and the $\left\{T_{i, j}\right\}$ with $i \neq j$ are $n^{2}-n$ independent variables [30, Theorem 12.4]. Thus $\operatorname{Mod}(F)$

is indeed isomorphic to $\mathbb{A}_{\mathbb{C}}^{n(n-1)}$. Further one observes that, in general, the matrix $L:=\left(T_{i, j}\right)$ has the property that $e^{2 \pi i L}$ is the topological monodromy at $z=0$ (or equivalently at $z=\infty$ ). Now, by the monodromy identity (Proposition 1), the entries of $e^{2 \pi i L}$ are (up to conjugation) rational in the $n(n-1)$-variables of Stokesmoduli $(F)$. This shows that there is no universal family above Stokesmoduli $(F)$.

In the case $n=2$, the $\operatorname{map} \operatorname{Mod}(F) \rightarrow \operatorname{Stokesmoduli}(F)$ can be made explicit and is shown to be surjective. For $n>2$, the above map is "highly transcendental" and we do not know whether it is surjective. The problem is the choice of a free vector bundle $\mathcal{M}$ in the definition of $\operatorname{Mod}(F)$. 
The problem of explicit computation of the Stokes matrices, i.e., making Stm explicit in this special case, has been studied over a long period and by many people G.D. Birkhoff, H. Turrittin, W. Balser, W.B. Jurkat, D.A. Lutz, K. Okubo, B. Dubrovin, D. Guzzetti et al. (see the introduction of [4] and also [5, 9, 15]). Using Laplace integrals the solutions of the above equation can be expressed in solutions of a 'transformed equation' with only regular singularities. The ordinary monodromy of the transformed equation produces answers for the Stokes matrices of the original equation. This is used by Dubrovin (see [10, Lemma 5.4, p. 97]), D. Guzzetti, H. Iritani, S. Tanabe, K. Ueda et al. in computations of the Stokes matrix for quantum differential equations, see [8, 9, 10, 15, 16, 25, 26, 27, 28].

Now we come to a surprising new case.

\subsection{Example: Totally ramified cases}

The formal data $F$ is essentially $V=V_{z^{1 / n}} \oplus V_{\zeta z^{1 / n}} \oplus \cdots \oplus V_{\zeta^{n-1} z^{1 / n}}$, where $\zeta:=e^{2 \pi i / n}$, each $V_{\zeta^{j} z^{1 / n}}$ has dimension 1 and $\gamma$ satisfies $\gamma^{n}=1$. The irregularity $\sum_{i \neq j} 1 \cdot 1 \cdot \operatorname{deg}\left(\zeta^{i} z^{1 / n}-\zeta^{j} z^{1 / n}\right)$ is equal to $n-1$ and this is small compared to the unramified case with irregularity $n(n-1)$. This is responsible for special features of these important examples.

For notational convenience we will consider the case $n=3$.

The lattice $N_{0}$ with formal data $F$ and trivial Stokes matrices can be represented by the differential operator $\delta+\left(\begin{array}{ccc}-\frac{1}{3} & 0 & z \\ 1 & 0 & 0 \\ 0 & 1 & \frac{1}{3}\end{array}\right)$. A computation (following [30, $\left.\S 12.5\right]$ ) shows that $\operatorname{Mod}(F)$ is represented by the universal family $\delta+\left(\begin{array}{ccc}a_{1} & 0 & z \\ 1 & a_{2} & 0 \\ 0 & 1 & a_{3}\end{array}\right)$ with $a_{1}, a_{2}, a_{3} \in \mathbb{C}$ with $a_{1}+a_{2}+a_{3}=0$.

There are 6 singular directions, corresponding to the differences of generalized eigenvalues $\zeta^{i} z^{1 / 3}-\zeta^{j} z^{1 / 3}$ for $i \neq j$. The corresponding Stokes matrix has one element off the diagonal, called $x_{j, i}$. Two singular directions are in $[0,1)$, namely $\frac{1}{4}, \frac{3}{4}$, and the others are obtained by shifts over 1 and 2. The topological monodromy at $z=0$ is conjugated (by the monodromy identity) to $\gamma \mathrm{St}_{3 / 4} \mathrm{St}_{1 / 4}$ which reads

$$
\left(\begin{array}{lll}
0 & 0 & 1 \\
1 & 0 & 0 \\
0 & 1 & 0
\end{array}\right)\left(\begin{array}{ccc}
1 & 0 & 0 \\
0 & 1 & 0 \\
0 & x_{2,1} & 1
\end{array}\right)\left(\begin{array}{ccc}
1 & x_{0,1} & 0 \\
0 & 1 & 0 \\
0 & 0 & 1
\end{array}\right)
$$

The characteristic polynomial of this matrix is $\lambda^{3}-x_{0,1} \lambda^{2}-x_{2,1} \lambda-1$. The topological monodromy is given by the operator $\delta+\left(\begin{array}{ccc}a_{1} & 0 & 0 \\ 1 & a_{2} & 0 \\ 0 & 1 & a_{3}\end{array}\right)$, which has eigenvalues $a_{1}, a_{2}, a_{3}$.

The monodromy has eigenvalues $e^{2 \pi i a_{j}}$ for $j=1,2,3$ and its characteristic polynomial is

$$
\left(\lambda-e^{2 \pi i a_{1}}\right)\left(\lambda-e^{2 \pi i a_{2}}\right)\left(\lambda-e^{2 \pi i a_{3}}\right) .
$$

Hence $x_{0,1}=\sum_{j=1}^{3} e^{2 \pi i a_{j}}$ and $x_{2,1}=\sum_{i<j} e^{2 \pi i a_{i}} e^{2 \pi i a_{j}}$. This makes the analytic morphism Stm from $\operatorname{Mod}(F)=\operatorname{Spec}\left(\mathbb{C}\left[a_{1}, a_{2}, a_{3}\right] /\left(a_{1}+a_{2}+a_{3}\right)\right)$ to $\operatorname{Stokesmoduli}(F)=\operatorname{Spec}\left(\mathbb{C}\left[x_{0,1}, x_{2,1}\right]\right)$ explicit.

Special cases:

(1) $a_{1}=-1 / 3, a_{2}=0, a_{3}=1 / 3$ yields $x_{0,1}=x_{2,1}=0$ and all Stokes matrices are trivial. 
(2) $a_{1}=a_{2}=a_{3}=0$ yields $x_{0,1}=\left(\begin{array}{l}3 \\ 1\end{array}\right), x_{2,1}=-\left(\begin{array}{l}3 \\ 2\end{array}\right)$. The equation is $\delta^{3}-z$ and all Stokes entries are \pm binomial coefficients.

For general $n, \operatorname{Mod}(F)$ is represented by the universal family

$$
\delta+\left(\begin{array}{ccccc}
a_{0} & 0 & \ddots & \ddots & z \\
1 & a_{1} & 0 & \ddots & 0 \\
\vdots & \vdots & \ddots & \ddots & \ddots \\
\cdot & \ldots & 1 & a_{n-2} & 0 \\
0 & \ldots & \ldots & 1 & a_{n-1}
\end{array}\right) \quad \text { with } \sum a_{j}=0
$$

The case $a_{i}=\frac{i}{n}-\frac{n-1}{2 n}$ for $i=0, \ldots, n-1$ corresponds to the case where all Stokes matrices are trivial.

The case all $a_{j}=0$ corresponds to $\delta^{n}-z$. For this equation, as in the case $n=3$, all the Stokes entries are \pm binomial coefficients. This is an explicit form of part of a conjecture of Dubrovin (see Section 4).

Conclusions. For the totally ramified cases $F$ that we consider, the Stokes matrices have explicit formulas in exponentials of algebraic expressions in the entries of the matrix differential operator. This shows in particular, that there is no universal family parametrized by $\operatorname{Stokesmoduli}(F)$. Further $\operatorname{Stm}: \operatorname{Mod}(F) \rightarrow \operatorname{Stokesmoduli}(F)$ is surjective and the fibers correspond to choices of the logarithm of the topological monodromy.

Also for a slightly more general case than the totally ramified case the entries the Stokes matrices are determined by the topological monodromy.

Proposition 2. Let $M$ be an irreducible differential module over the field $K=\mathbb{C}\left(\left\{z^{-1}\right\}\right)$. Suppose that the tuple $(V, \ldots)$, associated by Theorem 2 to $M$, has the properties:

(a) $V=V_{0} \oplus W$ where $V_{0}$ has eigenvalue $0, \operatorname{dim} V_{0}=m$, and $W$ is totally ramified, $\operatorname{dim} W=n$.

(b) The restriction of the formal monodromy $\gamma$ to $V_{0}$ has $m$ distinct eigenvalues.

Then, after normalization, the monodromy identity implies that the topological monodromy at $z=\infty$ determines all Stokes matrices.

Proof. The Malgrange irregularity $\operatorname{irr}(M)$ is $2 m+n-1$. Let $f_{1}, \ldots, f_{m}$ denote a basis of eigenvalues of $\gamma$ on $V_{0}$. Further we may suppose that $W=\oplus_{i=0}^{n-1} V_{\zeta^{i} z^{1 / n}}$, where $\zeta=e^{2 \pi i / n}$ and each $V_{\zeta^{i} z^{1 / n}}$ has dimension 1 . Let $e_{i}$ be a basis vector for $V_{\zeta^{i} z^{1 / n}}$ such that $\gamma e_{i}=e_{i+1}$ for $i=0, \ldots, n-2$ and $\gamma e_{n-1}=\lambda e_{0}$ for some $\lambda \in \mathbb{C}^{*}$.

The Stokes maps $\mathrm{St}_{d}$ for directions $d \in[0,1)$ are maps $\ell_{i}: V_{0} \rightarrow V_{\zeta^{i} z^{1 / n}}, \tilde{\ell}_{i}: V_{\zeta^{i} z^{1 / n}} \rightarrow V_{0}$ and $m_{i, j}: V_{\zeta^{i} z^{1 / n}} \rightarrow v_{\zeta^{j} z^{1 / n}}$ for $i \neq j$. Write $\ell_{i}\left(f_{j}\right)=x_{i, j} e_{i}, \tilde{\ell}_{i}\left(e_{i}\right)=\sum_{j} y_{j, i} f_{j}$ and $m_{i, j}\left(e_{i}\right)=z_{i, j} e_{j}$.

An element $c=\left(c_{1}, \ldots, c_{m}\right) \in\left(\mathbb{C}^{*}\right)^{m}$ acts on the basis $f_{1}, \ldots, f_{m}$ of $V_{0}$ by $f_{i} \mapsto c_{i} f_{i}$ for all $i$. Then $c$ acts on the Stokes entries by $x_{i, j} \mapsto c_{j} x_{i, j}, y_{j, i} \mapsto c_{j}^{-1} y_{j, i}$ and $z_{i, j} \mapsto z_{i, j}$.

Suppose that a $c=\left(c_{1}, \ldots, c_{m}\right) \neq 1$ acts trivially on the above Stokes entries. Then $c_{j} \neq 1$ implies $x_{i, j}=y_{j, i}=0$. Let $\tilde{V}_{0}$ be the subspace of $V_{0}$ generated by the $f_{j}$ with $c_{j}=1$. Then $\left(\tilde{V}_{0} \oplus W, \ldots\right)$ is a subobject of $(V, \ldots)$ and, as a consequence, $M$ is reducible.

Thus the action of $\left(\mathbb{C}^{*}\right)^{m}$ is faithful and one can produce a basis $f_{1}, \ldots, f_{m}$ such that $m$ of the Stokes entries are 1. This leaves $m+n-1$ unknown Stokes entries. The characteristic polynomial of the product $\gamma \prod_{d \in[0,1)} \mathrm{St}_{d}$ can be explicitly computed (compare [7]) and the entries of this polynomial are independent inhomogeneous linear expressions in the $z_{i, j}$ and the products $x_{i, j} y_{j, i}$. Thus the monodromy identity and normalizing some of the $x_{i, j}, y_{j, i}$ to 1 produces all $\mathrm{St}_{d}$ for $d \in[0,1)$. For general $d$, the Stokes matrix $\mathrm{St}_{d}$ is derived from the above, using the identity $\gamma^{-1} \mathrm{St}_{d} \gamma=\mathrm{St}_{d+1}$. 


\section{Fano varieties and quantum differential equations}

This part of the paper reviews work by J.A. Cruz Morales and the author [7]. A (complex) Fano variety $F$ is a non-singular, connected projective variety of dimension $d$ over $\mathbb{C}$, whose anticanonical bundle $\left(\Lambda^{d} \Omega\right)^{*}$ is ample. There are rather few Fano varieties.

Examples: For dimension 1 only $F=\mathbb{P}^{1}$; for dimension 2: the Fano's are del Pezzo surfaces and $\cong \mathbb{P}^{1} \times \mathbb{P}^{1}$ or $\cong$ to $\mathbb{P}^{2}$ blown up in at most 8 points in general position; for dimension 3 the list of Iskovshih-Mori-Mukai contains 105 deformation classes. According to Wikipedia, the classification of Fano varieties of higher dimension is a project called "the periodic table of mathematical shapes".

\subsection{Quantum cohomology and quantum differential equations}

We borrow from the informal introduction to the subject from M.A. Guest's book [12]. Let $F$ be a Fano variety. On the vector space $H^{*}(F):=\oplus_{i=0}^{d} H^{2 i}(F, \mathbb{C})$ there is the usual cup product - (say obtained by the wedge product of differential forms). Quantum cohomology introduces a deformation $\circ_{t}$ of the cup product $\circ$ on $H^{*}(F)$ for $t \in H^{2}(F, \mathbb{C})$.

With respect to a basis $b_{0}, \ldots, b_{s}$ of $H^{*}(F, \mathbb{Z}):=\oplus_{i=0}^{d} H^{2 i}(F, \mathbb{Z})$, the quantum products $b_{i} \circ_{t}$ have matrices which are computable in terms of the geometry of $F$. Let $b_{1}, \ldots, b_{r}$ be a basis of $H^{2}(F, \mathbb{Z})$.

The quantum differential equation of a Fano variety $F$ is a system of (partial) linear differential operators $\partial_{i}-b_{i} \circ_{t}, i=1, \ldots, r$ acting on the space of the holomorphic maps $H^{2}(F, \mathbb{C})=$ $\mathbb{C} b_{1}+\cdots+\mathbb{C} b_{r} \rightarrow H^{*}(F, \mathbb{C})=\mathbb{C} b_{0}+\cdots+\mathbb{C} b_{s}$.

For the case $r=1$ that interests us, the quantum differential equation reads $z \frac{d}{d z} \psi=C \psi$, where $\psi$ is a vector of lenght $s+1$ and $C$ is the matrix of quantum multiplication $b_{1} \circ_{t}$. The entries of the $(s+1) \times(s+1)$ matrix $C$ are polynomials in $z$ with integer coefficients. Clearly $z=0$ is a regular singular point and $z=\infty$ is irregular singular. By taking a cyclic vector one obtains a scalar differential equation of order $s+1$.

\subsection{Examples of quantum differential equations}

Below we present examples of quantum differential equations.

$\delta^{n}-z$ with $\delta=z \frac{d}{d z}$ for $\mathbb{P}^{n-1}$.

$\delta^{n+m-1}-m^{m} z\left(\delta+\frac{m-1}{m}\right)\left(\delta+\frac{m-2}{m}\right) \ldots\left(\delta+\frac{1}{m}\right)$ with $n \geq 1, m>1$ for a non-singular hypersurface of degree $m$ in $\mathbb{P}^{n+m-1}[12, \S 3.2$, Example 3.6, p. 43].

$\prod_{j=0}^{n} \delta\left(\delta-\frac{1}{w_{j}}\right) \cdots\left(\delta-\frac{w_{j}-1}{w_{j}}\right)-z$ for the weighted projective space $\mathbb{P}\left(w_{0}, \ldots, w_{n}\right)$ (an orbifold).

$\delta^{3}-a z \delta^{2}-\left((b-a) z^{2}+b z\right) \delta+2 a z^{2}-c z^{3}$ with $a, b, c \in \mathbb{Z}$ for del Pezzo surfaces.

$\delta^{4}-11 z \delta^{2}-11 z \delta-3 z-z^{2}$ for $V_{5}$, for a linear section of the Grasmannian $G(2,5)$ in the Plücker embedding.

$\delta^{4}-\left(94 z^{2}+6 z\right) \delta^{2}-\left(484 z^{3}+188 z^{2}+2 z\right) \delta-\left(695 z^{4}+632 z^{3}+98 z^{2}\right)$ for the 3-fold $V_{22}$.

B. Dubrovin is one of the founders of quantum cohomology. One of his conjectures $[9,10]$ states that the Gram matrix $\left(G_{i, j}\right)$ of a (good) Fano variety coincides with the "Stokes matrix" of the quantum differential equation of $F$ (up to a certain equivalence of matrices).

Here $G_{i, j}=\sum_{k}(-1)^{k} \operatorname{dim} \operatorname{Ext}^{k}\left(\mathcal{E}_{i}, \mathcal{E}_{j}\right)$, where $\left\{\mathcal{E}_{i}\right\}$ is an exceptional collection of coherent sheaves on $F$ generating the derived category $D^{b} \operatorname{coh}(F)$. Further "Stokes matrix" is in fact a connection matrix and, in our terminology, equal to the product $\prod_{d \in[0,1 / 2)} \mathrm{St}_{d}$ (in counter clock order).

For $F=\mathbb{P}^{n}$, this has been verified by D. Guzzetti [15]. There are recent papers $[13,14,16$, 21, 25, 26, 27, 28] which handle more cases, e.g., Calabi-Yau complete intersections in weighted 
projective spaces, Grassmanians, cubic surfaces, toric orbifolds. They use the "Fourier-Laplace type transformation" to a regular singular differential equation, mentioned in Section 3.1.

The contribution of [7] is proving Dubrovin's conjecture by computing all $\mathrm{St}_{d}$, using only the formal classification and the monodromy identity, for the cases $\mathbb{P}^{n}$, non singular hypersurfaces of degree $m \leq n$ in $\mathbb{P}^{n}$, and for weighted projective spaces $\mathbb{P}\left(w_{0}, \ldots, w_{n}\right)$. The equations and the method are closely related to Sections 2.5 and 3.2.

\section{Riemann-Hilbert approach to Painlevé equations}

This classical method, related to isomonodromy, was revived and refined by M. Jimbo, T. Miwa and K. Ueno [17, 18]. The literature on the subject is nowadays impressive.

Certain details of the Riemann-Hilbert approach are worked out in collaboration with M.-H. Saito [29]. In collaboration with J. Top, refined calculations of Okamoto-Painlevé spaces

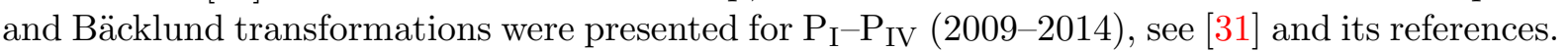
We give here a rough sketch of the ideas and especially of the part where Stokes matrices enter the picture. The starting point is a 'family' $\mathbf{S}$ of differential modules $M$ over $\mathbb{C}(z)$ with prescribed singularities at fixed points of $\mathbb{P}^{1}$. A priori $\mathbf{S}$ is just a set. One side of the Riemann-Hilbert approach is to construct a moduli space $\mathcal{M}$ over $\mathbb{C}$ such that $\mathbf{S}$ has a natural identification with $\mathcal{M}(\mathbb{C})$. This part does not involve Stokes maps.

The other side of the Riemann-Hilbert approach is a "monodromy space" $\mathcal{R}$ built out of monodromy, Stokes matrices and 'links'. The space $\mathcal{R}$ is determined by the prescribed type and position of the singularities of $\mathbf{S}$.

An example for $\mathcal{R}$. The set $\mathbf{S}$ which gives rise to $\mathrm{P}_{\mathrm{III}}(D 7)$ consists of the differential modules $M$ over $\mathbb{C}(z)$ which have only 0 and $\infty$ as singular points. The point 0 has Katz invariant $1 / 2$ and the point $\infty$ has Katz invariant 1 (see Section 2.2 for the definition of the Katz invariant). The monodromy space $\mathcal{R}$ consists of the analytic classification $(V(0), \ldots)$ of $M$ at $z=0$ and $(V(\infty), \ldots)$ at $z=\infty$ and a connection matrix between these data, the link $L: V(0) \rightarrow V(\infty)$, which describes the relation between the solutions around $z=0$ and the solutions around $z=\infty$.

The solution space $V(0)$ at $z=0$ is given a basis $e_{1}, e_{2}$ for which the formal monodromy, the Stokes matrix and topological monodromy top are $_{0}$

$$
\left(\begin{array}{cc}
0 & -1 \\
1 & 0
\end{array}\right), \quad\left(\begin{array}{ll}
1 & 0 \\
e & 1
\end{array}\right), \quad\left(\begin{array}{cc}
-e & -1 \\
1 & 0
\end{array}\right)
$$

The solution space $V(\infty)$ at $z=\infty$ is given a basis $f_{1}, f_{2}$ for which the formal monodromy, the Stokes maps and the topological monodromy top $\infty_{\infty}$ are

$$
\left(\begin{array}{cc}
\alpha & 0 \\
0 & \alpha^{-1}
\end{array}\right), \quad\left(\begin{array}{cc}
1 & 0 \\
c_{1} & 1
\end{array}\right), \quad\left(\begin{array}{cc}
1 & c_{2} \\
0 & 1
\end{array}\right), \quad\left(\begin{array}{cc}
\alpha & \alpha c_{2} \\
\alpha^{-1} c_{1} & \alpha^{-1}\left(1+c_{1} c_{2}\right)
\end{array}\right) .
$$

One may assume that $L:=\left(\begin{array}{cc}\ell_{1} & \ell_{2} \\ \ell_{3} & \ell_{4}\end{array}\right)$ has determinant 1 . There is a relation top ${ }_{0} \cdot L^{-1} \cdot \operatorname{top}_{\infty} \cdot L=$ 1. This yields a set of variables and relations and thus an affine variety. The above bases $e_{1}, e_{2}$ of $V(0)$ and $f_{1}, f_{2}$ of $V(\infty)$ are not unique. Indeed, the ambiguity in these basis is given by the transformation $e_{1}, e_{2} \mapsto \lambda_{0} e_{1}, \lambda_{0} e_{2}$ and $f_{1}, f_{2} \mapsto \lambda_{1} f_{1}, \lambda_{2} f_{2}$ with $\left(\lambda_{0}, \lambda_{1}, \lambda_{2}\right) \in\left(\mathbb{C}^{*}\right)^{3}$. By dividing this affine space by the action of $\left(\mathbb{C}^{*}\right)^{3}$ one obtains $\mathcal{R}$. The final result is that $\mathcal{R}$ is an affine cubic surface, given by variables $\ell_{13}, \ell_{23}, \alpha$ and relation $\ell_{13} \ell_{23} e+\ell_{13}^{2}+\ell_{23}^{2}+\alpha \ell_{13}+\ell_{23}=0$.

Consider the map $\mathbf{S} \rightarrow \mathcal{R}$ which associates to each module $M \in \mathbf{S}$ its monodromy data in $\mathcal{R}$. The fibers of this map are parametrized by some $T \cong \mathbb{C}^{*}$ and there results a bijection $\mathbf{S} \rightarrow \mathcal{R} \times T$. The set $\mathbf{S}$ has a priori no structure of an algebraic variety. A moduli space $\mathcal{M}$ over $\mathbb{C}$, whose set of closed points consists of certain connections of rank two on the projective 
line, is constructed such that $\mathbf{S}$ coincides with $\mathcal{M}(\mathbb{C})$. This defines the analytic RiemannHilbert morphism $\mathrm{RH}: \mathcal{M} \rightarrow \mathcal{R}$. The fibers of $\mathrm{RH}$ are the isomonodromic families. There results an extended Riemann-Hilbert isomorphism $\mathrm{RH}^{+}: \mathcal{M} \rightarrow \mathcal{R} \times T$. From the isomorphism $\mathrm{RH}^{+}$the Painlevé property for the corresponding Painlevé equation follows and the moduli space $\mathcal{M}$ is identified with an Okamoto-Painlevé space. Special properties of solutions of the Painlevé equations, such as special solutions, Bäcklund transformations etc., are derived from the extended Riemann-Hilbert isomorphism.

The above sketch needs subtle refinements. One has, depending on the Painlevé equation and its parameters, to add level structure, to forget points, to desingularize $\mathcal{R}$ and $\mathcal{M}$, to replace spaces by their universal covering etc., in order to obtain a correct extended Riemann-Hilbert isomorphism. For the remarkable fact that for each Painlevé equation the moduli space for the monodromy $\mathcal{R}$ is an affine cubic surface with three lines at infinity, there is not yet an explanation.

\section{Acknowlegdements}

The author likes to thank the referees for their work and the very useful comments which led to many improvements.

\section{References}

[1] Babbitt D.G., Varadarajan V.S., Local moduli for meromorphic differential equations, Astérisque (1989), $1-217$.

[2] Balser W., From divergent power series to analytic functions. Theory and application of multisummable power series, Lecture Notes in Math., Vol. 1582, Springer-Verlag, Berlin, 1994.

[3] Balser W., Formal power series and linear systems of meromorphic ordinary differential equations, Universitext, Springer-Verlag, New York, 2000.

[4] Balser W., Jurkat W.B., Lutz D.A., On the reduction of connection problems for differential equations with an irregular singular point to ones with only regular singularities. I, SIAM J. Math. Anal. 12 (1981), 691-721.

[5] Balser W., Jurkat W.B., Lutz D.A., On the reduction of connection problems for differential equations with an irregular singular point to ones with only regular singularities. II, SIAM J. Math. Anal. 19 (1988), 398-443.

[6] Cano J., Ramis J.-P., Théorie de Galois différentielle, multisommabilité et phénomenes de Stokes, in preparation.

[7] Cruz Morales J.A., van der Put M., Stokes matrices for the quantum differential equations of some Fano varieties, Eur. J. Math. 1 (2015), 138-153, arXiv:1211.5266.

[8] Dubrovin B., Geometry of 2D topological field theories, in Integrable Systems and Quantum Groups (Montecatini Terme, 1993), Lecture Notes in Math., Vol. 1620, Springer, Berlin, 1996, 120-348, hep-th/9407018.

[9] Dubrovin B., Geometry and analytic theory of Frobenius manifolds, in Proceedings of the International Congress of Mathematicians, Vol. II (Berlin, 1998), Doc. Math. (1998), Extra Vol. II, 315-326, math.AG/9807034.

[10] Dubrovin B., Painlevé transcendents in two-dimensional topological field theory, in The Painlevé Property, CRM Ser. Math. Phys., Springer, New York, 1999, 287-412, math.AG/9803107.

[11] Duval A., Mitschi C., Matrices de Stokes et groupe de Galois des équations hypergéométriques confluentes généralisées, Pacific J. Math. 138 (1989), 25-56.

[12] Guest M.A., From quantum cohomology to integrable systems, Oxford Graduate Texts in Mathematics, Vol. 15, Oxford University Press, Oxford, 2008.

[13] Guest M.A., Its A., Lin C.-S., Isomonodromy aspects of the $t t^{*}$ equations of Cecotti and Vafa I. Stokes data, arXiv:1209.2045.

[14] Guest M.A., Sakai H., Orbifold quantum D-modules associated to weighted projective spaces, Comment. Math. Helv. 89 (2014), 273-297, arXiv:0810.4236. 
[15] Guzzetti D., Stokes matrices and monodromy of the quantum cohomology of projective spaces, Comm. Math. Phys. 207 (1999), 341-383, math.AG/9904099.

[16] Iritani H., An integral structure in quantum cohomology and mirror symmetry for toric orbifolds, $A d v$. Math. 222 (2009), 1016-1079, arXiv:0903.1463.

[17] Jimbo M., Miwa T., Ueno K., Monodromy preserving deformation of linear ordinary differential equations with rational coefficients. I. General theory and $\tau$-function, Phys. D 2 (1981), 306-352.

[18] Jimbo M., Miwa T., Monodromy preserving deformation of linear ordinary differential equations with rational coefficients. II, Phys. D 2 (1981), 407-448.

[19] Loday-Richaud M., Stokes phenomenon, multisummability and differential Galois groups, Ann. Inst. Fourier (Grenoble) 44 (1994), 849-906.

[20] Loday-Richaud M., Pourcin G., On index theorems for linear ordinary differential operators, Ann. Inst. Fourier (Grenoble) 47 (1997), 1379-1424.

[21] Marcolli M., Tabuada G., From exceptional collections to motivic decompositions via noncommutative motives, J. Reine Angew. Math. 701 (2015), 153-167, arXiv:1202.6297.

[22] Mitschi C., Differential Galois groups of confluent generalized hypergeometric equations: an approach using Stokes multipliers, Pacific J. Math. 176 (1996), 365-405.

[23] Ramis J.-P., Stokes phenomenon: historical background, in The Stokes Phenomenon and Hilbert's 16th Problem (Groningen, 1995), World Sci. Publ., River Edge, NJ, 1996, 1-5.

[24] Stokes G.G., Early letters to lady Stokes, March 17, 1857, in Memoir and Scientific Correspondence of the Late Sir George Gabriel Stokes, Bart, Cambridge University Press, Cambridge, 2010, 50-76.

[25] Tanabé S., Invariant of the hypergeometric group associated to the quantum cohomology of the projective space, Bull. Sci. Math. 128 (2004), 811-827, math.AG/0201090.

[26] Tanabé S., Ueda K., Invariants of hypergeometric groups for Calabi-Yau complete intersections in weighted projective spaces, Commun. Number Theory Phys. 7 (2013), 327-359, arXiv:1305.1659.

[27] Ueda K., Stokes matrices for the quantum cohomologies of Grassmannians, Int. Math. Res. Not. 2005 (2005), 2075-2086, math.AG/0503355.

[28] Ueda K., Stokes matrix for the quantum cohomology of cubic surfaces, math.AG/0505350.

[29] van der Put M., Saito M.-H., Moduli spaces for linear differential equations and the Painlevé equations, Ann. Inst. Fourier (Grenoble) 59 (2009), 2611-2667, arXiv:0902.1702.

[30] van der Put M., Singer M.F., Galois theory of linear differential equations, Grundlehren der Mathematischen Wissenschaften, Vol. 328, Springer-Verlag, Berlin, 2003.

[31] van der Put M., Top J., Geometric aspects of the Painlevé equations PIII(D $\left.\mathrm{D}_{6}\right)$ and PIII( $\left.\mathrm{D}_{7}\right)$, SIGMA 10 (2014), 050, 24 pages, arXiv:1207.4023. 